\title{
Therapeutic groups via video teleconferencing and the impact on group cohesion
}

\author{
Amy Lopez ${ }^{1}$, Brian Rothberg ${ }^{2}$, Emily Reaser $^{3}$, Sarah Schwenk $^{1}$, Rachel Griffin ${ }^{1}$ \\ ${ }^{1}$ Helen and Arthur E. Johnson Depression Center, ${ }^{2}$ Department of Psychiatry, University of Colorado Anschutz Medical Campus, Aurora, CO, \\ USA; ${ }^{3}$ Graduate School of Social Work, University of Denver, Denver, CO, USA \\ Contributions: (I) Conception and design: A Lopez, E Reaser; (II) Administrative support: E Reaser; (III) Provision of study materials or patients: A \\ Lopez, S Schwenk, R Griffin; (IV) Collection and assembly of data: A Lopez, E Reaser; (V) Data analysis and interpretation: A Lopez, S Schwenk, R \\ Griffin; (VI) Manuscript writing: All authors; (VII) Final approval of manuscript: All authors. \\ Correspondence to: Amy Lopez, PhD, LCSW. Helen and Arthur E. Johnson Depression Center, University of Colorado Anschutz Medical Campus, \\ 13199 E. Montview Blvd., Aurora, CO 80045, USA Email: Amy.lopez@cuanschutz.edu.
}

Background: The use of synchronous telemental health for therapy group is a relatively new area of exploration. While telegroups have demonstrated they can be helpful in providing psychoeducation, there is less known about whether synchronous remote groups can develop group cohesion and whether patients can receive the benefit of a supportive group setting.

Methods: A pilot study was conducted comparing group cohesion between patients who participated in a dialectical behavior therapy (DBT) group via video teleconferencing (VTC) and patients who participated in an in-person group.

Results: Findings indicate that while both groups felt equally connected to the facilitator, there were significant differences between the online and in-person groups on the group cohesion scale. Those in the online group did not feel as connected to other group members as those in the in-person group. Qualitative statements indicate that while an in-person group may have been preferable, the convenience of the online group outweighed any negative effects. Attendance was significantly better in the online group, suggesting that use of this technology may help to overcome barriers preventing treatment participation. The focus of this study was on group cohesion and changes in psychiatric symptoms were not evaluated.

Conclusions: The use of telemental health for group therapy appears to be a viable alternative to traditional in-person groups, especially when no other treatment options are available. However, facilitators may need to take extra steps to build group cohesion when members are participating remotely.

Keywords: Online therapy groups; video teleconferencing (VTC) groups; online dialectical behavior therapy (DBT) groups; group cohesion; telemental health

Received: 13 August 2019; Accepted: 12 November 2019; Published: 05 April 2020.

doi: $10.21037 /$ mhealth.2019.11.04

View this article at: http://dx.doi.org/10.21037/mhealth.2019.11.04

\section{Introduction}

Improving access to appropriate treatment is a concern for many mental health practitioners. Telemental health, herein defined as any technology-based interventions, including synchronous video conferencing, interactive computer programs or apps, text messaging, e-mails, and self-guided computer programs, has been offered as one solution to increase access by mitigating barriers that often prevent patients from participating in mental health treatment (1). A dialectical behavior therapy (DBT) group was offered via video teleconferencing (VTC) to increase patient access to evidence-based treatments. This group was developed in response to patient request, primarily from those who wanted to participate in DBT but cited significant barriers 
preventing them from attending the group in person. The need to create the online group came from four different patient categories, based on barrier to care, including patients who:

(I). Lived locally but could not attend due to work;

(II). Had transportation difficulties;

(III). Had other commitments, such as child-care responsibilities, that made it difficult to attend an in-person group;

(IV). Lived in rural communities where this treatment is not offered.

A synchronous telehealth group was offered via VTC as an option to mitigate the above barriers. There is very limited evidence for the efficacy or feasibility of a synchronous group via telehealth (2). However, literature indicates that psychoeducational groups offered via telehealth allow for patients to learn new information (3), although there remains some question as to whether patients can also receive the supportive benefit of being in group with others when they are participating through an online platform rather than in person. Group cohesion is a key component of successful group therapy (4) and there is also a need to explore the impacts of telehealth delivery on group connection and whether telehealth allows for the same or comparable benefits of mutual support inherent in traditional face-to-face group therapy settings.

This paper explores a small pilot study comparing group experience and group cohesion between those patients who participated in an online group and those who participated in an in-person group. Findings from this study indicate that developing group cohesion via telehealth is possible and offers some lessons learned to inform those who wish to provide synchronous group therapy via telehealth.

\section{Literature review}

Although telehealth is becoming more common in mental health practices, the use of group therapy via telehealth has not been as widely studied. Most of the previous studies of telehealth and group therapy focus on asynchronous groups, such as chat rooms or message boards. These studies have demonstrated that people can connect in group settings remotely and find ways to support each other despite the distance and communication limitations (5). Research has demonstrated that online groups follow similar patterns of development, including getting to know each other, creation of group norms, providing mutual support, and challenging each other to grow (6). The research has found some commonalities of the most effective online asynchronous groups. First, the groups that had a trained facilitator had better outcomes than those that were peer-led. Second, the groups that had a structured psychoeducational component were also found to be more effective than those that were more general conversation around a common topic (6). These studies have provided a foundation of evidence that remote asynchronous groups are a feasible option for providing patients education and support remotely (2).

While the literature is relatively robust as to asynchronous groups, chat rooms, and message boards, there is less information about the use of interactive or synchronous groups. Groups that utilize message boards and chat rooms allow only for expression of emotion via written posts, which then limits verbal intonation, body language, and other emotional indicators that allow patients to build and deepen emotional bonds in the group (4). Theories of social presence would suggest that by adding in the video and audio cues to the group interactions, the level of feelings of connection would increase, which would likely enhance the level of support in the group setting (7). However, much of this is still theory as the impacts of increased communication cues on group experience are not yet fully explored in the literature. Researchers have begun to understand how these additional cues may influence group connection and support in a telehealth environment, specifically those groups conducted via VTC. Recent studies have begun to evaluate not only the effectiveness of the treatment, but also whether the additional audio and visual cues provided through VTC can compensate for the feelings of distance due to remote participation. (5).

One study explored the use of VTC groups in which a remote therapist facilitated the group from a different location than the members of the group. In this approach, the group members were all at the same location, but the group was facilitated by a trained mental health professional located at a remote site (8). Evaluation of the group found that the group members had an improvement of symptoms, but there were questions about the group cohesion. Specifically, findings indicate the group felt connected to each other and developed traditional group process, but that they did not feel connected to the remote facilitators.

A slightly different type of VTC group is one that is more psychoeducational, but where the researcher and the participants are all participating via VTC. Researchers conducted a study on an online mindfulness group, with the focus of the group on learning mindfulness skills and their application rather than on building a supportive 
community. The participants were given mindfulness CD's, asked to practice at home, and then tasked with reporting their findings back during the group sessions. Findings indicate that $85 \%$ percent of the participants felt that they had increased their learning about mindfulness and $81 \%$ reported satisfaction with the group itself. Qualitative statements indicated that while they might have preferred to learn the mindfulness in person, access to the CD's between sessions and the convenience and flexibility of the online group outweighed any negative effects of not being able to meet in person (9).

Although the VTC option has more evidence for use with psychoeducational groups where learning is the focus, many questions remain as to how this method might work for a traditional supportive therapy group. A study was conducted with a support group for caregivers of patients with dementia (10). The group members and facilitators were all separately located and participated in a facilitated weekly group via VTC. Findings from the study indicate that the group was able to develop cohesion and feelings of support. However, they also found the need for some modifications in their interactions in order to better support each other when social cues were not available in the same way, such as clearly stating their emotions explicitly rather than relying on implicit cues. The group members shared that although they would have preferred to be in an inperson group, the nature of their caregiving would not allow for it. Without the VTC option, they would receive no support at all. Despite the preference for in-person group, the remote group still allowed for participation in a group they could not otherwise have, while also providing a supportive and rewarding group experience (10).

Although there is some foundation for use of group in telehealth settings, there is a need for further exploration of synchronous groups and the use of VTC for group treatment. Because group cohesion is a primary factor in a successful group experience, further research is needed to explore how use of telehealth delivery may affect this group process. To better understand the role of telehealth and group therapy, this pilot study provided synchronous, VTC telehealth groups and then evaluated how the technology influenced group cohesion and connection.

\section{Methods}

The study was conducted at a University-based mental health clinic that is open to the community at large. At the time of this study, the clinic was offering two weekly DBT skills training groups: an online group and an in-person group. It was determined through consultation with the University IRB that because the clinic already offered the groups as part of existing programming this was considered a clinic quality improvement evaluation rather than a new research study. As such, IRB approval was not necessary.

Dialectical Behavioral Therapy is a well-researched treatment with strong outcomes and has some initial research that treatment outcomes are similar when the DBT skills training is provided in online formats $(11,12)$. DBT offers a combination of psychoeducational curriculum and a supportive component to the group, and was therefore considered a good treatment for this pilot study. The online group used the same format, the same materials, and had the same facilitator as the in-person group, with the only difference being the manner in which the materials were presented. Both groups were led by a licensed mental health provider who has been intensively trained to provide DBT, and has been leading DBT skills groups for over 10 years.

\section{Design}

The pilot study used a post-only two group comparison design. Patients self-selected into the group format that worked best for them (telegroup or in-person). Data was collected at completion of all the modules of the DBT group, with telegroup completing an electronic based survey and the in-person group completing a hard copy. Participation in the survey was completely voluntary for both groups, and patients could opt out of completing the survey. Each survey included the group scale, as well as satisfaction questions, and three open-ended survey questions to further elaborate on the group experience.

\section{Participants}

Thirty-five patients participated, with 15 people who received the treatment via online groups and 20 people who received the DBT via in-person groups. Despite the self-selection process, the two groups were similar in demographics and composition. Average age for both groups was 40.7 with a range of 22-70 years for the telegroup (SD $=16.1)$ and $20-66$ for the in-person group $(\mathrm{SD}=15.1)$. The telegroup was $73 \%$ female compared to $70 \%$ female for the in-person group. Race was mostly Caucasian in both groups, with only one person of color in each group. Depression was the primary diagnosis in both groups, but each group also had those with primary diagnoses of bipolar disorder and 
Table 1 Final means and SDs of the Rovai scale

\begin{tabular}{lccc}
\hline Group type & Facilitator relationship subscale score & Group member role subscale* & Total scale score* $^{*}$ \\
\hline Telegroup (mean, SD) & $14.1(1.5)$ & $20.3(2.4)$ & $35.3(3.9)$ \\
In-Person Group (mean, SD) & $14.7(1.0)$ & $23.6(1.7)$ & $40.5(2.8)$ \\
\hline
\end{tabular}

*, differences: $P<0.001$.

anxiety. Statistical analyses found no significant differences in age, gender, race, or diagnosis between the two groups.

\section{Measures}

Group experience was measured using the Rovai Community Connection Scale (13). This measure is adapted from online education programs and focuses on the feelings of connection within a community. The Rovai scale has questions related to learning of the therapeutic concepts, connection with the facilitator, role as a group member, and how the participants feel about their interactions with each other. The questions were rated on a 1-5 Likert scale, with higher scores indicating higher levels of connection. This measure was chosen over other group cohesion scales because of its demonstrated effectiveness to capture feelings of connection in both in-person and virtual settings (13). The measure has two subscales, one focusing on community and member interaction the second focusing on learning and the relationship with the facilitator. These two scales were then combined into a total score to capture the group experience as a whole. Scale reliabilities were conducted, with all of the scales falling within acceptable ranges of alpha levels of 0.8 or higher. Changes in mental health outcomes were not assessed as the primary focus was on group experience. In addition to the survey, group attendance, which is also a measure of therapeutic engagement (14), was compared between the two groups. Attendance data was collected from the clinic's electronic medical record.

\section{Results}

\section{Rovai scale}

Differences in scale and subscale scores between the two groups were analyzed using an independent samples $t$-test. The mean scores for the facilitator subscale were 14.1 (SD $=1.5$ ) for the telegroup and $14.7(\mathrm{SD}=1.0)$ for the in-person group. There were no significant differences between the two groups on the facilitator subscale $(t=1.2, \mathrm{P}=0.21)$, indicating that the groups felt similarly about their ability to learn the skills and their relationship with the facilitator.

However, there were significant differences between the groups on the other scales. On the member subscale, the tele group had a mean score of $20.3(\mathrm{SD}=2.4)$ and the inperson group had a mean score of $23.6(\mathrm{SD}=1.7)$. This was a statistically significant difference $(t=3.6, \mathrm{P}<0.001)$, with the online group reporting lower levels of connection to other members. On the total scale, the mean score for the telegroup was 35.3 ( $\mathrm{SD}=3.9$ ) and the mean score for the in-person group was $40.5(\mathrm{SD}=2.8)$. This was a statistically significant difference on the combined scale $(t=3.7$, $\mathrm{P}<0.001)$, with the telegroup reporting less group cohesion than the in-person group (Table 1).

\section{Patient satisfaction}

As a measure of patient satisfaction, the participants were asked, on a scale of $1-5$, how likely they would be to recommend this type of treatment to a friend. In the online group, the mean score was $4.5(\mathrm{SD}=0.73)$ and the mean score for the in-person group was $4.8(\mathrm{SD}=0.88)$ This was not a statistically significant difference $(t=1.39, \mathrm{P}=0.19)$.

\section{Attendance}

The attendance rates for each group were calculated by dividing the number of enrolled participants with the number who attended each week. The telegroup had an average attendance rate of $91 \%$, whereas the in-person group had an average attendance rate of $75 \%$. This was a statistically significant difference $(t=2.64, \mathrm{P}<0.01)$ showing greater average attendance for the telegroup.

\section{Qualitative statements}

Group members were given the option to provide additional 
qualitative feedback about the group experience. The inperson group offered a few suggestions about the content of the group, such as the need to provide more practice time and wanting more specific examples of how the skills could be used. The comments from the telegroup were more directly related to the group process. There were comments about the need to better explain the features of the telehealth program and frustrations from members about background noise and other distractions. Overall, comments from the telegroup members suggested that despite any concerns the format allowed them the opportunity to participate in a group they might not otherwise be able to do. Some comments from participants in the telegroup include:

* "I think the format is great and has helped me immensely. I think the group participation is somewhat lacking since the computer format provides another subtle barrier to communication. For me, it's allowed me to participate and learn. I think the facilitator has been great in working with this system."

* "I really enjoyed this and overall learned a lot. I did not however feel very connected to the group given the format it was challenging to be in connection with folks."

* "For my purposes, the online sessions were a God-send. I think the recent inclusion of break-out sessions belped promote more interaction and communication by team members and to bring a sense of community to the online sessions. There are probably other improvements that can be identified, but the growth I saw from my start to my finish has me convinced that this is a legitimate format for folks that have other commitments."

* "It is kind of odd not meeting in person, but the breakout rooms help to overcome the awkwardness. The online meetings make it logistically possible for me to participate easily."

\section{Discussion}

This pilot study demonstrates that use of telehealth for group therapy may be an option for providing mental health treatment for those who have significant barriers to participating in traditional face-to-face treatment. The analysis from the Rovai scale indicates that the relationship with the facilitator and how members felt about their learning was similar between the two groups. Both groups were equally as satisfied with the group experience, with no statistically significant differences between the two groups.
Despite the virtual environment, the members were still able to benefit from learning the DBT skills. This may be an indicator that for psychoeducation groups, manualized based evidence-based treatments, or other types of groups in which the learning of skills is the focus, an online/ telehealth group may be a comparable treatment option to an in-person group. This is supported by research conducted by Gros et. al, who found that Evidence based programs presented online have comparable outcomes to those delivered in person (11).

However, there was a significant difference on the Rovai scale between the two groups in relation to member interaction and group cohesion. The results indicate that the group members found that it was harder to connect with each other in the virtual environment. This is not an unusual phenomenon even in traditional group development processes. Group members tend to first connect with the leader, with the allegiance shifting to the group as it develops (4). It is possible that the data collection was done too early in the group process, especially when also introducing a new technology. There is ongoing need to tease this out in the literature, but until that is possible, those wanting to provide an online group may want to consider what types of groups are most appropriate for an online delivery. Groups that rely more on member to member interaction, such as support groups or interpersonal/relationship groups, may prove to be more challenging. However, exploration of the different features of the teleconferencing program may offer some ways to overcome these barriers to create community and build relationships between the group members. For example, the breakout rooms seemed to help with patient interaction. After the introduction of the breakout room feature in the teleconferencing program, the patients reported in their qualitative statements that using the breakout rooms to allow them more time to interact with each other made a difference in how they felt about the group as a whole.

Previous qualitative studies (8-10) have demonstrated that while an in-person group may have been preferable, due to personal barriers, attending an in-person group was not an option. The qualitative statements from this study had similar sentiments. In each of these studies, including this one, the convenience of the telehealth group outweighed any negative benefits of not meeting in person. In fact, for most of these participants, if they had not done the telegroup, they would not have been able to do any DBT group at all. While attending the group via telehealth 
would not have been their first choice, telehealth treatment was preferable to no treatment at all.

One other finding of importance relates to the attendance. Those in the telegroup had a statistically significantly better attendance rate, with most of members attending every single session. Although an in-person group may have allowed for more group interaction, many of the in-person participants had to miss due to other obligations. The analysis of the attendance rate is not only an indicator that people were engaged enough to attend regularly, it likely also demonstrates that it was easier for those in the telehealth group to overcome barriers that often prevent patients from participating in treatment.

While further research is needed, this study provides a foundation to support telegroup as a noninferior treatment for those who cannot participate in a traditional face-toface setting. In some cases, telehealth group may be the only way in which patients can access treatment, creating a viable alternative for those who face treatment barriers. While telehealth groups can provide a comparable group experience, some modifications may need to be made to help build group cohesion and create an experience that meets patients' therapeutic needs.

\section{Lessons learned for facilitation of online groups}

\section{Technology use}

The members of the group had varying experience with technology, ranging from those who used technology regularly as part of their job to those who had no previous experience with video conferencing. In order to address this, each participant was offered a "practice session" with the clinic administrative staff prior to the start of group. This practice session allowed them to explore the features of the program in a non-therapeutic environment and with only one other person rather than in front of the entire group. Many of the members turned down the practice session, stating they felt comfortable enough with the program to be able to navigate it well. If the members opted not to do a practice session, they were still sent information on how to contact a live person during the time of the group in case they ran into any technical difficulties.

Previous studies of telemental health have shown that patients have decreased satisfaction with VTC when the facilitator/clinician is not comfortable with the technology (15). To address this the facilitator also used practice sessions prior to introducing any new feature. Working with other clinic staff, the facilitator had the chance to see how the technology would work, get feedback about the use of it, and talk through the best way to explain the different features to the group members. Many different VTC features were used regularly and became essential to group facilitation to either enhance learning, build connection, or encourage participation from less active members, including:

(I). The white board for drawing or writing;

(II). Screen sharing of picture or other mindfulness activities;

(III). Chat (typing responses);

(IV). Breakout rooms.

\section{Speaking}

Because there are not the typical social cues it becomes more difficult to know when to talk or speak freely when using the online platform. Some of the ways in which this was addressed through this pilot stage was by using the technology itself to enhance the process. Unlike in-person groups, where members may be able to speak at will or go in a specific order based on where they are sitting, in this setting, the facilitator would call on people or direct the order in which participants should speak. While not ideal, the group members also used other techniques to get the attention of the group, such as raising of hands - either through the technology or by physically raising their hands. This group began using other non-verbal signals as a way to show support (thumbs up), to disagree (thumbs down), or nodding of the heads in agreement. The group also used the chat feature regularly as a way to share their opinion without an interruption to the flow of the conversation. In fact, when they were asked to use the chat feature for certain activities, those members who rarely spoke interacted more. When this was discovered, the facilitator regularly incorporated chat into the activities as a way to provide those with different communication styles the opportunity to practice the skills.

\section{Distractions and background}

One of the primary complaints from the members was background noise and other interruptions. Although the facilitator frequently reminded people to turn on their mute buttons when not speaking to reduce background noise, members often forgot. The facilitator began muting people to avoid this problem, but then when people would try to speak, they could not be heard as they were muted. This created some disruption in the flow of the conversation, but 
it was typically not enough of a problem to distract from the conversation as a whole or cause frustration among the members.

In addition to the noise, there were a number of other distractions within people's personal environments, such as pets walking into the screen, ceiling fans spinning, and even a member holding a sleeping baby. Most of these distractions were met with good humor and the group was able to move forward. In some cases, these background events helped the members get to know each other better by being able experience the members private environments in a way that is not possible in traditional group settings.

\section{Privacy}

Privacy was another concern to be considered. Because of the nature of the group setting, privacy is already somewhat compromised, but becomes more so when members are all participating in remote settings where the environment cannot be controlled by the facilitator. Prior to the start of the group, the members agreed that they would meet only in places that were private to avoid non-group members seeing the group members on their screen. The group discussed the importance of recognizing that they could be compromising the privacy of other patients if they were not careful about the environment in which they were participating in the group. Through the course of the study, the members were mindful about this, with most participating from their homes, private offices at their jobs, or even from a parked car outside their work.

There was a request that the sessions be recorded so that those who miss group could view the lesson later. While being able to access the group lesson asynchronously is a benefit to using technology, there was not a way that the research team could store and share these video recordings that was also HIPAA compliant and protected patient privacy. This is an ongoing discussion and a consideration for future groups as it is a significant benefit to be able to provide the same lessons asynchronously to those who may not be able to attend regularly or may want to review the lessons on their own.

\section{Building community}

One of the challenges of providing groups in this format was how to build community and cohesion among people who were not in the same physical space. This was done doing traditional methods (introductions, ice breakers, finding commonalities, etc.) but there were also ways in which building community was unique to this setting. One example is the formal use of group member's names when speaking. With in-person groups, learning each other's names can often take time. However, using video conferencing, all of the members' names show up underneath their picture-almost like a name tag. The technology allows for the members to set their usernames based on what they would like to be called. This allowed the group to reference each other and speak to each other using names, rather than pointing or using pronouns as can happen with in-person groups.

A second example of using the technology to build community is use of "breakout rooms." This feature allows for two or more members to videoconference, rather than being part of the full group. This feature was used during the Interpersonal Effectiveness module, which allowed the members of the group to practice their interpersonal skills with one other person. The feedback from use of this feature was good, with members sharing how it allowed them the chance to get to know others better. The members enjoyed this process so much, it has now been incorporated into the other modules.

Beyond the intentional use of technology, group leaders may need to take the lead initially on helping the group learn to interact with each other in the virtual environment. As the group norming process develops, the group should also be encouraged to talk about what is (and is not working) for their interactions, both as a way to clarify the expectations, but also to help build a sense of community by working together to find ways to interact successfully. Further research likely could provide clarity as to the skills a facilitator may need to employ in order to help build group cohesion in the virtual environment similar to how facilitators help build the group norms in traditional group settings.

\section{Limitations}

As this pilot study helps demonstrate, use of telehealth for group therapy does allow for the benefits of group connection, but there is still much that needs to be explored. This study has several limitations that will need to be considered in future research. First, this study had a small sample size and ongoing research is necessary to determine whether these findings could be replicated with larger samples. This was also primarily a convenience sample and may not apply to a broader population. The clients were not randomized, but this was in part because most of those who 
picked the telegroup needed it for specific reasons. In fact, most of them would not have been able to participate at all if the telegroup had not been offered. While randomization may be beneficial in the future, it should not limit the ability of those who want to participate, but cannot. Finally, the primary question remains as to whether these findings would be applicable to more traditional group therapy settings. Although the DBT has a supportive component to it, the focus is primarily on learning the skills. It is not clear from this study as to whether the VTC technology would be appropriate to use in group settings, such as groups that are more supportive in nature or psychodynamically oriented.

\section{Conclusions}

The use of VTC and telehealth for group therapy may be a possible treatment option for those facing treatment barriers. While it may not be preferential, it does allow for a treatment option when none exists. This study also suggests that the facilitators will need to have awareness around how to develop group norms online and ways in which technology can be used intentionally to try and increase connection and support among group members.

\section{Acknowledgments}

The authors would like to thank the Helen and Arthur E. Johnson Depression Center board and donors for their ongoing philanthropic support.

Funding: None.

\section{Footnote}

Conflicts of Interest: The authors have no conflicts of interest to declare.

Ethical Statement: The authors are accountable for all aspects of the work in ensuring that questions related to the accuracy or integrity of any part of the work are appropriately investigated and resolved. This study was considered a clinic quality improvement evaluation rather than a new research study, IRB approval was not necessary.

Open Access Statement: This is an Open Access article distributed in accordance with the Creative Commons Attribution-NonCommercial-NoDerivs 4.0 International License (CC BY-NC-ND 4.0), which permits the noncommercial replication and distribution of the article with the strict proviso that no changes or edits are made and the original work is properly cited (including links to both the formal publication through the relevant DOI and the license). See: https://creativecommons.org/licenses/by-nc$\mathrm{nd} / 4.0 \%$.

\section{References}

1. Yellowlees P, Shore JH. Telepsychiatry and health technologies: a guide for mental health professionals. First edition. Arlington: American Psychiatric Pub, 2018.

2. Banbury A, Nancarrow S, Dart J, et al. Telehealth Interventions Delivering Home-based Support Group Videoconferencing: Systematic Review. J Med Internet Res 2018;20:e25

3. Barak A, Boniel-Nissim M, Suler J. Fostering empowerment in online support groups. Comput Human Behav 2008;24:1867-83.

4. Yalom ID, Leszcz M. The theory and practice of group psychotherapy. 5th ed. New York: Basic Books, 2005.

5. Berger $\mathrm{T}$. The therapeutic alliance in internet interventions: A narrative review and suggestions for future research. Psychother Res 2017;27:511-24.

6. Eysenbach G, Powell J, Englesakis M, et al. Health related virtual communities and electronic support groups: systematic review of the effects of online peer to peer interactions. BMJ 2004;328:1166 .

7. Rettie R. Mobile Phone Communication: Extending Goffman to Mediated Interaction. Sociology 2009;43:421-38.

8. Greene CJ, Morland LA, Macdonald A, et al. How does tele-mental health affect group therapy process? Secondary analysis of a noninferiority trial. J Consult Clin Psychol 2010;78:746-50.

9. Niles BL, Klunk-Gillis J, Ryngala DJ, et al. Comparing mindfulness and psychoeducation treatments for combatrelated PTSD using a telehealth approach. Psychol Trauma 2012;4:538-47.

10. O'Connell ME, Crossley M, Cammer A, et al. Development and evaluation of a telehealth videoconferenced support group for rural spouses of individuals diagnosed with atypical early-onset dementias. Dementia (London) 2014;13:382-95.

11. Gros DF, Morland LA, Greene CJ, et al. Delivery of Evidence-Based Psychotherapy via Video Telehealth. J Psychopathol Behav Assess 2013;35:506-21.

12. Wilks CR. Internet-Delivered Dialectical Behavior Therapy Skills Training for Suicidal and Heavy 
Drinkers. Dissertation for PhD Degree. University of Washington, 2018.

13. Rovai AP. Development of an instrument to measure classroom community. Internet High Educ 2002;5:197-211.

14. Reardon ML, Cukrowicz KC, Reeves MD, et. al. Duration and Regularity of Therapy Attendance as Predictors of

doi: $10.21037 / \mathrm{mhealth} .2019 .11 .04$

Cite this article as: Lopez A, Rothberg B, Reaser E, Schwenk S, Griffin R.Therapeutic groups via video teleconferencing and the impact on group cohesion. mHealth 2020;6:13.
Treatment Outcome in an Adult Outpatient Population. Psychother Res 2002;12:273-85.

15. Farabee D, Calhoun S, Veliz R. An Experimental Comparison of Telepsychiatry and Conventional Psychiatry for Parolees. Psychiatr Serv 2016;67:562-5. 